\title{
Quantum Hall Effect of Massless Dirac Fermions in a Vanishing Magnetic Field
}

\author{
Kentaro Nomura, ${ }^{1}$ Shinsei Ryu, ${ }^{2}$ Mikito Koshino, ${ }^{3,4}$ Christopher Mudry, ${ }^{5}$ and Akira Furusaki ${ }^{6}$ \\ ${ }^{1}$ Department of Physics, Tohoku University, Sendai, 980-8578, Japan \\ ${ }^{2}$ Kavli Institute for Theoretical Physics, University of California, Santa Barbara, California 93106, USA \\ ${ }^{3}$ Department of Physics, Tokyo Institute of Technology, Meguro-ku, Tokyo 152-8551, Japan \\ ${ }^{4}$ Physics Department, Columbia University, New York, New York 10027, USA \\ ${ }^{5}$ Condensed Matter Theory Group, Paul Scherrer Institute, CH-5232 Villigen PSI, Switzerland \\ ${ }^{6}$ Condensed Matter Theory Laboratory, RIKEN, Wako, Saitama 351-0198, Japan
}

(Received 20 January 2008; published 20 June 2008)

\begin{abstract}
The effect of strong long-range disorder on the quantization of the Hall conductivity $\sigma_{x y}$ in graphene is studied numerically. It is shown that increasing Landau-level mixing progressively destroys all plateaus in $\sigma_{x y}$ except the plateaus at $\sigma_{x y}=\mp e^{2} / 2 h$ (per valley and per spin). The critical state at the Dirac point is robust to strong disorder and belongs to the universality class of the conventional plateau transitions in the integer quantum Hall effect. We propose that the breaking of time-reversal symmetry by ripples in graphene can realize this quantum critical point in a vanishing magnetic field.
\end{abstract}

DOI: 10.1103/PhysRevLett.100.246806

Graphene, an isolated layer of graphite [1], displays a remarkable quantization of the Hall conductivity $\sigma_{x y}$ when subjected to a magnetic field. As shown in Refs. [2,3], $\sigma_{x y}$ is measured to be either a negative or a positive half-integer $n+1 / 2$ in units of $4 e^{2} / h$. This should be contrasted with the usual integer quantum Hall effect (IQHE) for which quantum Hall plateaus of the Hall conductance in a $\mathrm{Si}$ metal oxide semiconductor field-effect transistor or in GaAs/AlGaAs heterojunctions occur at positive integer values $n$ in units of $e^{2} / h$ [4]. In the vicinity of the two nonequivalent corners $K$ and $K^{\prime}$ of the first Brillouin zone of graphene, the noninteracting electronic dispersion is linear; i.e., at the so-called Dirac point it realizes a noninteracting massless Dirac Hamiltonian. As the Hall conductivity of a massive two-component Dirac fermion in $(2+1)$-dimensional space and time has long been known to be a half-integer in units of $e^{2} / h[5,6]$, the observed quantization of the Hall conductivity of graphene can be attributed to four independent flavors of two-component Dirac fermions with the spin-degeneracy accounting for two and the valley-degeneracy accounting for another two flavors.

This explanation fails to account for Anderson localization due to disorder in graphene, as recently emphasized in Ref. [7]. Disorder can introduce both intervalley and intravalley scattering between the Bloch states in the valleys centered at $K$ and $K^{\prime}$ [8-10]. The physics of localization for the Bloch (Landau) states of graphene is predicted to depend sensitively on the nature of the disorder and, in particular, on the range of the spatial correlations of the impurities. When disorder is short-ranged, intervalley scattering has large matrix elements. In this case, all Bloch states are localized at zero magnetic field [8], while the IQHE is wiped out by a sufficiently strong disorder [9]. On the other hand, when disorder is sufficiently long-ranged, intervalley scattering is negligible, and the metallic phase
PACS numbers: 73.43.-f, 72.10.-d, 73.21.-b, 73.50.Fq

remains stable to disorder if time-reversal symmetry is present [11-14].

As for the conventional IQHE, the quantization of the Hall conductivity in graphene requires the existence of at least one critical state in each impurity-broadened Landau level (LL) induced by a magnetic field. The destruction of the quantum Hall plateaus in the conventional IQHE with increasing disorder strength $[15,16]$ can be understood in terms of a critical state in a disorder-broadened LL migrating (levitating) to higher filling fraction as soon as the disorder induces significant LL mixing [17]. The same phenomenon has been shown to be operative for all impurity-broadened LLs in graphene when the disorder is short-ranged [9].

In this Letter, we argue on the basis of numerical calculation that, when the disorder is sufficiently long-ranged, all but one critical states in graphene undergo levitation as the disorder strength increases. The exception is the state at the Dirac point that remains critical whatever the disorder strength is. Consequently, graphene with long-range disorder has the remarkable property that the quantum Hall plateaus with $\sigma_{x y}= \pm e^{2} / 2 h$ per spin and per valley survive in the limit of strong disorder. In the language of the renormalization group, the scaling flows of the longitudinal $\left(\sigma_{x x}\right)$ and transverse $\left(\sigma_{x y}\right)$ conductivities for graphene with strong infinite-range disorder are determined by two attractive fixed points at $\sigma_{x y}= \pm e^{2} / 2 h$ and one repulsive fixed point at $\sigma_{x y}=0$. For comparison, the corresponding phase diagram for graphene with strong short-range disorder is conventional in that it is characterized by a single attractive fixed point, the insulating phase with $\sigma_{x x}=$ $\sigma_{x y}=0[9,10]$. Of course, the range of the disorder is always finite in a sample of graphene [18]. However, the characteristic scattering length induced by short-range impurities could be longer than the phase coherence length 
for some range of temperature. Indeed it has been found, on a sample of graphene with a high mobility $(\mu \simeq 2 \times$ $10^{4} \mathrm{~cm}^{2} / \mathrm{V} \mathrm{s}$ ), that the longitudinal conductivity at the Dirac point at zero magnetic field never falls with decreasing temperature down to $10 \mathrm{mK}$ [19]; i.e., intervalley scattering is irrelevant in this range of temperature.

The honeycomb lattice of graphene has two atoms per unit cell on sites labeled $A$ and $B$. Linearization of the noninteracting lattice Hamiltonian in the clean limit, ignoring the spin degrees of freedom, yields [1]

$$
\begin{aligned}
\mathcal{H} & :=\left(\begin{array}{cc}
\mathcal{H}_{K} & 0 \\
0 & \mathcal{H}_{K^{\prime}}
\end{array}\right), \\
\mathcal{H}_{K} & =\boldsymbol{v}_{F} \boldsymbol{\sigma} \cdot[-i \hbar \nabla+e \boldsymbol{A}],
\end{aligned}
$$

where $\mathcal{H}_{K^{\prime}}$ is the transpose of $\mathcal{H}_{K}, v_{F}$ is the Fermi velocity at the Dirac point, and the Pauli matrices $\boldsymbol{\sigma}$ act on the sublattice degrees of freedom. In a finite magnetic field $\boldsymbol{B}=\boldsymbol{\nabla} \times \boldsymbol{A}=(0,0, B)$ the spectrum of $\mathcal{H}_{K}$ consists of LLs with eigenvalues $E_{n}=\operatorname{sgn}(n) \hbar \omega_{0} \sqrt{|n|}$ with $n \in \mathbb{Z}$ [20]. The energy and length scales are set by $\omega_{0}=$ $\sqrt{2} v_{F} / \ell_{B}$ and $\ell_{B}=\sqrt{\hbar / e B}$. The filling fraction $\nu>0$ $(\nu<0)$ is the ratio of the number of occupied (empty) states above (below) the Dirac point to the LL degeneracy.

In this work, as we neglect intervalley and spindependent scattering channels, every LL is fourfold degenerate. The problem of Anderson localization that we shall consider is defined by the random Dirac equation $\left[\mathcal{H}_{K}+V(\boldsymbol{r})\right] \psi=\varepsilon \psi$ with the static vector potential in the Landau gauge $\boldsymbol{A}=(0, B x, 0)$ and $V$ a random static scalar potential. We choose two-dimensional space to be a square with area $L^{2}$. We introduce a cutoff $n_{c}=9$ for the LLs. The disorder potential is $V(\boldsymbol{r})=\sum_{j=1}^{N_{\text {imp }}} u_{j} \exp (-\mid \boldsymbol{r}-$ $\left.\left.\boldsymbol{R}_{j}\right|^{2} / 2 d^{2}\right) /\left(2 \pi d^{2}\right)$ with $\boldsymbol{r} \in \mathbb{R}^{2}, d$ a length scale, and $u / d^{2}=\left|u_{j}\right| / d^{2}$ an energy scale. The signs in $u_{j}= \pm u$ and the scattering centers $\boldsymbol{R}_{j}$ are chosen randomly and independently at $N_{\text {imp }}$ locations. The disorder strength is estimated to be the broadening of the LLs $\Gamma^{2} / 4=$ $2 \pi u^{2} N_{\text {imp }} /\left[\left(\ell_{B}^{2}+2 d^{2}\right) L^{2}\right]$ within the self-consistent Born approximation [15]. In the following we set $d=0.7 l_{B}$.

We evaluate the transverse conductivity with the Kubo formula

$$
\sigma_{x y}=-\frac{\hbar e^{2}}{L^{2}} \sum_{\varepsilon_{m}<\varepsilon_{F}<\varepsilon_{n}} \overline{\left(\frac{\operatorname{Im}\left[\left\langle m\left|v_{x}\right| n\right\rangle\left\langle n\left|v_{y}\right| m\right\rangle\right]}{\left(\varepsilon_{m}-\varepsilon_{n}\right)^{2}}\right)},
$$

where $|n\rangle$ denotes an eigenstate with its eigenvalue $\varepsilon_{n}$ of Hamiltonian $\mathcal{H}_{K}+V(\boldsymbol{r})$, the corresponding velocity operator is $\boldsymbol{v}:=\boldsymbol{v}_{F} \boldsymbol{\sigma}$, and the overline represents disorder averaging. The dependence of the disorder-average Hall conductivity $\sigma_{x y}$ on the filling fraction is shown in Fig. 1 for three values of the dimensionless disorder strength. For small LL mixing induced by the disorder, we observe that the curve $(a)$ has plateaus centered at half-integer values and with rounded corners up to $\nu= \pm\left(n_{c}+1 / 2\right)$. There are fewer well-defined Hall plateaus at moderately strong

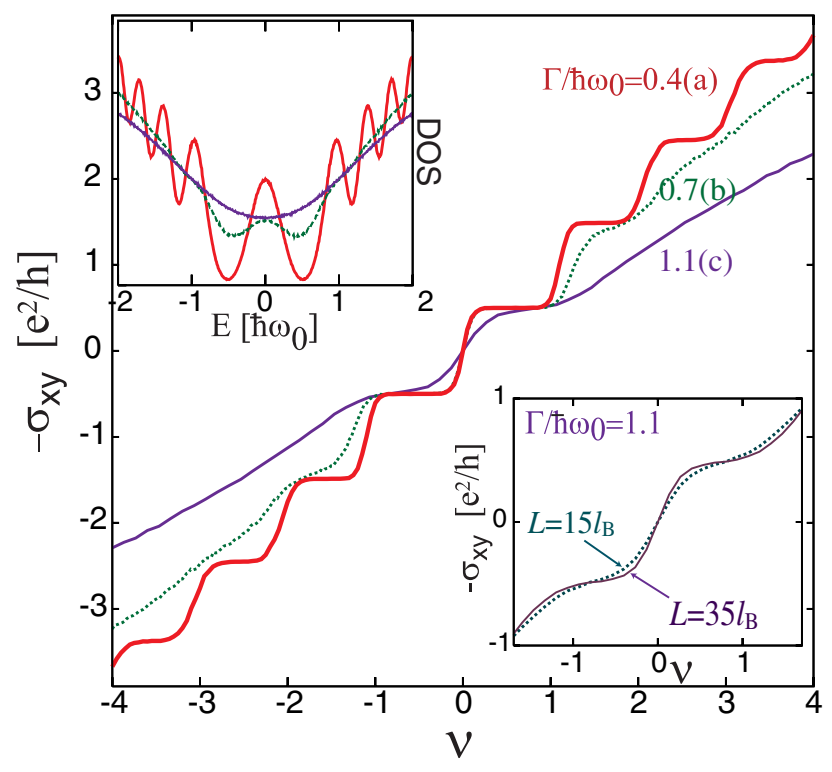

FIG. 1 (color online). Disorder-average Hall conductivity $\sigma_{x y}$ as a function of the filling fraction $\nu$ for the dimensionless disorder strength (a) $\Gamma / \hbar \omega_{0}=0.4$, (b) 0.7 , and (c) 1.1. Upper inset: Disorder-average density of states (DOS). Lower inset: Size dependence of $\sigma_{x y}$ at $\Gamma / \hbar \omega_{0}=1.1$.

disorder strength as shown in the curve $(b)$. Only the plateaus centered at $\nu= \pm 1 / 2$ are seen in the curve $(c)$ when the disorder is strong relative to $\hbar \omega_{0}$. The size dependence of $\sigma_{x y}$ at strong disorder (c) shown in the lower inset of Fig. 1 is consistent with the expectation that $\sigma_{x y}$ has a step at $\nu=0$ in the $L \rightarrow \infty$ limit. The quantization of $\sigma_{x y} /\left(e^{2} / h\right)$ for all half-integers larger in magnitude than $1 / 2$ correlates with an oscillatory dependence on the energy of the disorder-average density of states (DOS) as is illustrated with the upper inset of Fig. 1. This correlation is not present for the plateaus at $\nu= \pm 1 / 2$. LL mixing induced by the disorder is thus responsible for the disappearance of all Hall plateaus with $\left|\sigma_{x y}\right|>e^{2} / 2 h$ while the plateaus at $\nu= \pm 1 / 2$ are robust to strong disorder.

We now turn our attention to the dissipative component of electrical transport by studying the Thouless number $g_{T} \equiv\langle|\Delta \varepsilon|\rangle / \delta \varepsilon$. The Thouless energy $\Delta \varepsilon$ is the energy shift induced on energy eigenvalues by using antiperiodic instead of periodic boundary conditions [15]. The mean level spacing is $\delta \varepsilon=1 /\left(L^{2} \overline{\rho(\varepsilon)}\right)$ with $\rho(\varepsilon)$ the DOS. The angular brackets denote the typical value $\langle\Delta \varepsilon\rangle \equiv$ $\exp (\overline{\ln \Delta \varepsilon})$. The dependence of $g_{T}$ on $\nu$ at different values of $L / \ell_{B}$ is shown in Fig. 2 for weak (a), moderate (b), and strong (c) disorder strength. We observe a decrease of the Thouless number with the deviations of the filling fraction from the integer values $n$, which is well correlated with the oscillatory dependence of the DOS in Fig. 1. This decrease gets more pronounced the closer $n$ is to $n=0$. Local minima of the Thouless number are reached at half-integer filling fractions. At fixed filling fraction, $g_{T}$ generally 


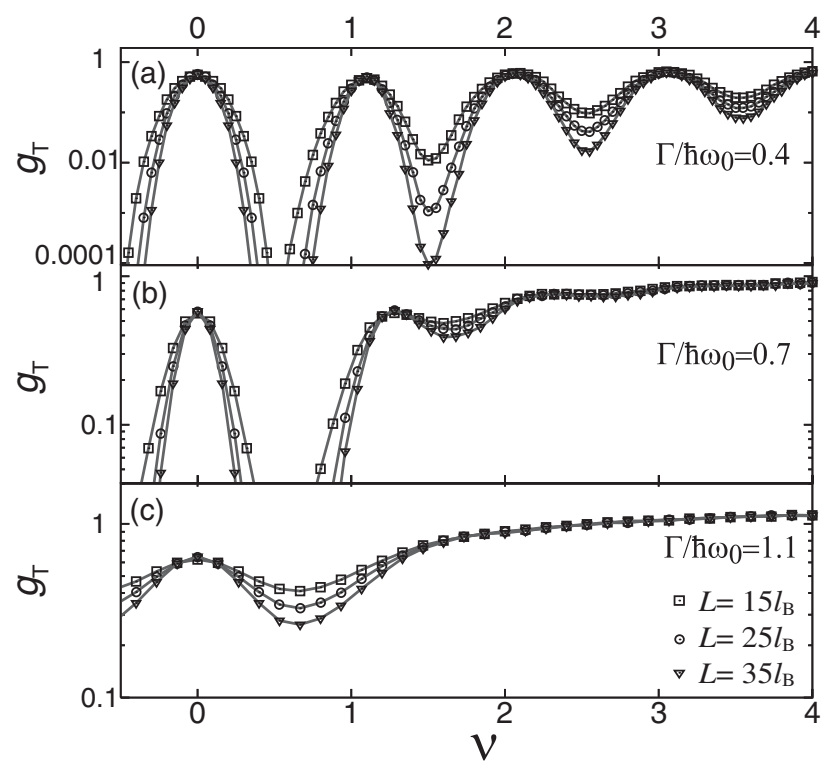

FIG. 2. The Thouless conductance $g_{T}$ as a function of the filling fraction $\nu$ when (a) $\Gamma / \hbar \omega_{0}=0.4$, (b) 0.7 , and (c) 1.1 , for various system sizes, $L / \ell_{B}=15(\square), 25(\bigcirc)$, and $35(\nabla)$.

decreases as the system size $L / \ell_{B}$ increases, while only at the center of an impurity-broadened LL it is independent of $L / \ell_{B}$. This is consistent with the existence of a critical state close to integer filling fractions. The decrease of $g_{T}$ gets more pronounced when approaching half-integer filling fractions. Increasing disorder strength as shown in Figs. 2(b) and 2(c) weakens the dependence of $g_{T}$ on both $\nu$ and $L / \ell_{B}$ around all half-integer filling fractions larger than $1 / 2$. A decrease of $g_{T}$ remains clearly visible at $\nu=1 / 2$ for strong disorder.

For any filling fraction, a localization length $\xi$ is extracted from the fit $g_{T}(L)=g_{0} \exp (-L / \xi)$ and $1 / \xi$ is plotted in Fig. 3(a) for weak, moderate, and strong disorder. Figure 3(a) is consistent with an oscillatory dependence of $\xi$ on $\nu$ that correlates with the DOS in Fig. 1 and also shows that $\xi$ increases for $\nu>1$ with disorder strength $\Gamma /\left(\hbar \omega_{0}\right)$. The latter feature is familiar in the conventional IQHE, where the LL mixing makes the localization weaker in the high-field regime [15]. In each impurity-broadened LL of Fig. 3(a), there exists a $\nu$ for which $\xi$ takes a maximum and much larger value than $L$. This $\nu$ corresponds to a local maximum of $g_{T}$ and to a critical singleparticle state. In the limit of no LL mixing, critical states are found at integer values of $\nu$. Figure 3(b) shows that all but one critical states are increasing functions of $|\nu|$ as the amount of LL mixing induced by the disorder increases. The exception is the $\nu=0$ critical state that remains pinned to the Dirac point for all disorder strength in Fig. 3(b). Consequently in the weak magnetic field limit $\hbar \omega_{0} / \Gamma \rightarrow 0$ all single-particle states in the hole (electron) regime are localized: $\sigma_{x y} /\left(e^{2} / h\right)=-\frac{1}{2}\left(+\frac{1}{2}\right)$ with the step discontinuity in $\sigma_{x y}$ occurring at $\nu=0$. This behavior is different from the one for the conventional IQHE systems
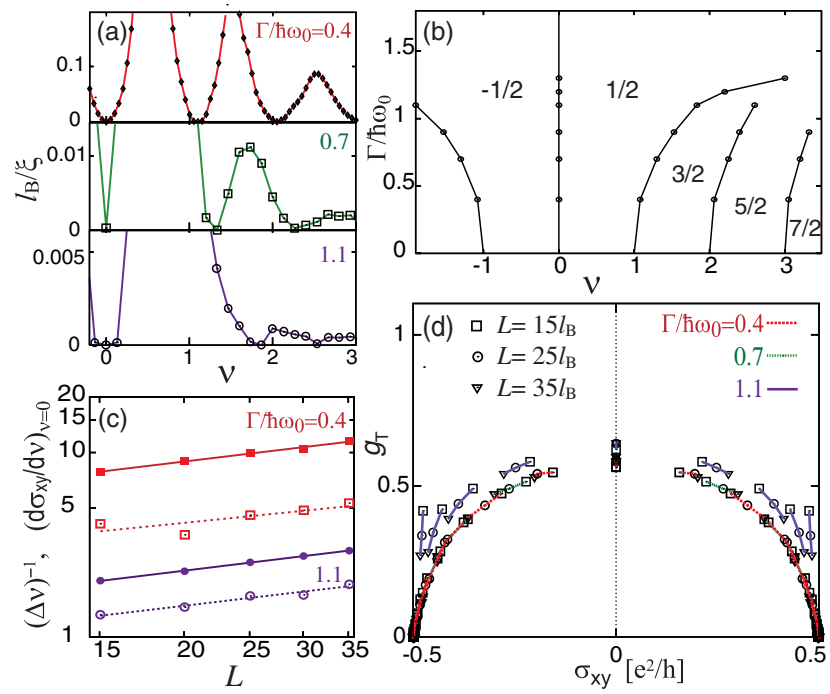

FIG. 3 (color online). (a) Dependence on $\nu$ of the inverse localization length $1 / \xi$. (b) The phase diagram for the quantum Hall effect of massless Dirac fermions. For each impuritybroadened LL, the phase boundaries are identified from the $L$-independent $g_{T}$ (circles). Half-integers indicate $-\sigma_{x y} /\left(e^{2} /\right.$ $h$ ). (c) Widths of the half-maxima of the Thouless conductance (closed symbols) and the slope of the Hall conductivity (open symbols) as functions of $L$. (d) Scaling flow in $\sigma_{x y}-g_{T}$ plane: $\sigma_{x y}$ and $g_{T}$ at $L / \ell_{B}=15(\square), 25(\bigcirc)$, and $35(\nabla)$ are plotted.

where $\sigma_{x y} \rightarrow 0$ (Hall insulator) in the weak magnetic field limit $[15,16]$. It is also different from the one observed in numerical studies of the effects of short-range correlated disorder on the IQHE in graphene [9], which reported strong localization behavior in the vicinity of $\nu=0$ over a wide range of magnetic field. This difference can be used to distinguish whether the dominant source of disorder in graphene is short- or long-range. For $\nu$ close to $\nu=0$ and from weak to strong disorder, Fig. 3(c) is consistent with the scaling ansatz $\sigma_{x y}=f_{x y}\left(L^{1 / \alpha_{\nu}} \nu\right)$ and $g_{T}=f_{x x}\left(L^{1 / \alpha_{\nu}} \nu\right)$ with $\alpha_{\nu} \approx 2$.3. Correspondingly, $1 / \xi \sim|\nu|^{\alpha_{\nu}}, 1 /\left(\Delta_{T} \nu\right) \sim$ $L^{\alpha_{\sigma}}$ where $\Delta_{T} \nu$ is the half-width of the peak of $g_{T}$ at $\nu=$ 0 , and $d \sigma_{x y} / d \nu \sim L^{\alpha_{\sigma}}$ with $\alpha_{\sigma}=1 / \alpha_{\nu}$. Moreover, $g_{T}=$ $f_{x x}\left(f_{x y}^{-1}\left(\sigma_{x y}\right)\right)$ must hold close to $\nu=0$, a prediction confirmed by the scaling flow in Fig. 3(d), where $\left(\sigma_{x y}, g_{T}\right)$ computed at $L=15,25$, and 35 are seen to move towards the attractive fixed points at $\left( \pm e^{2} / 2 h, 0\right)$ with increasing $L$.

The phase boundary separating the quantum Hall phases $\sigma_{x y}=-e^{2} / 2 h$ from $\sigma_{x y}=+e^{2} / 2 h$ in Fig. 3(b) extends to the zero-magnetic field limit $\hbar \omega_{0} / \Gamma \rightarrow 0$. This strongly suggests that the Dirac point is never localized by longrange disorder, however strong the disorder is. The Dirac point of graphene with long-range disorder remains the critical point of the IQHE plateau transition for any weak magnetic field.

We close this Letter by studying the effects of effective time-reversal symmetry breaking random perturbation on the zero-magnetic field limit $\hbar \omega_{0} / \Gamma \rightarrow 0$ of the Dirac 


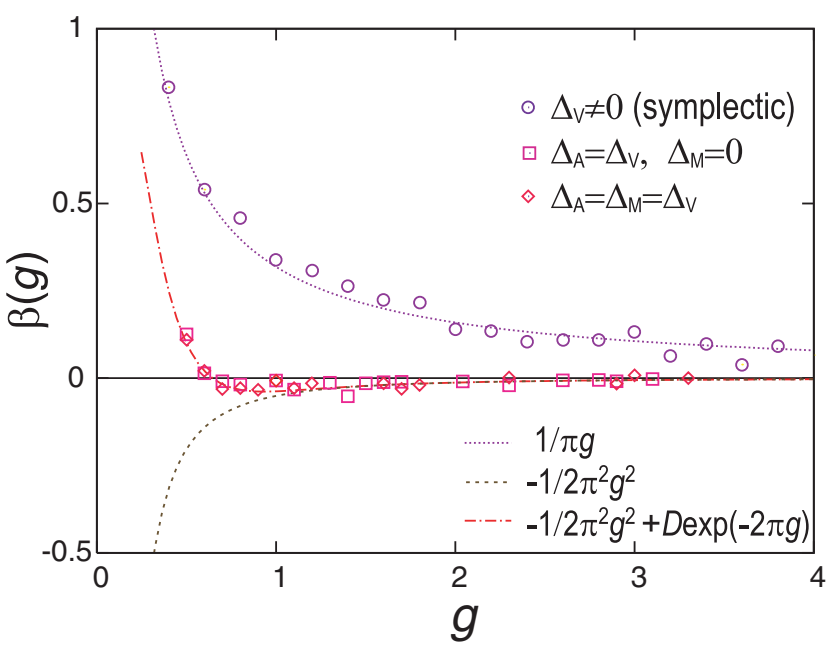

FIG. 4 (color online). The $\beta$ functions for the random Dirac Hamiltonians (3); $\beta(\mathrm{g})$ is independent of the mean value of $V$.

Hamiltonian (1). The random single-particle Dirac Hamiltonian is [6]

$$
\mathcal{H}:=\boldsymbol{\sigma} \cdot\left[-i \hbar v_{F} \boldsymbol{\nabla}+\boldsymbol{a}(\boldsymbol{r})\right]+m(\boldsymbol{r}) \sigma_{z}+V(\boldsymbol{r}),
$$

where $\boldsymbol{a}(\boldsymbol{r}), m(\boldsymbol{r})$, and $V(\boldsymbol{r})$ are Gaussian distributed with the covariances $\overline{f_{\mu}(\boldsymbol{q}) f_{\nu}\left(\boldsymbol{q}^{\prime}\right)}=\delta_{\mu \nu} \Delta_{\mu} \exp \left(-\boldsymbol{q}^{2} d^{2} / 2\right) \delta(\boldsymbol{q}+$ $\left.\boldsymbol{q}^{\prime}\right)$, if $\left(f_{\mu=0,1,2,3}\right)=\left(V, a_{1}, a_{2}, m\right)$ whereby $\Delta_{\mu}=\Delta_{V}$ for $\mu=0, \Delta_{\mu}=\Delta_{A}$ for $\mu=1,2$, and $\Delta_{\mu}=\Delta_{M}$ for $\mu=3$. The length scale $d$ is the range of the potential that varies up to $1 / 30$ of the minimal system size. The "random vector potential" $\boldsymbol{a}(\boldsymbol{r})$ describes the effect of ripples in corrugated graphene [21,22]. Ludwig et al. in Ref. [6] conjectured that the random Dirac Hamiltonian (3) flows into the critical point describing the quantum Hall plateau transition. Using the numerical method from Ref. [14], we present in Fig. 4 the $\beta$ function $\beta(g)$ for the Kubo conductivity $g \equiv \sigma_{x x} /\left(e^{2} / h\right)$ which we fit against $\beta(g)=$ $-1 /\left(2 \pi^{2} g^{2}\right)+D \exp (-2 \pi g)$ [4] whenever time-reversal symmetry is broken $\left(-i \sigma_{y} \mathcal{H}^{*} i \sigma_{y} \neq \mathcal{H}\right)$ by either $\Delta_{A}>$ 0 or $\Delta_{M}>0$. The fitting parameter $D$ is optimized when $D=7$. Figure 4 is consistent with a fixed point at $g \simeq 0.6$ which is attractive in $g$. The $\beta$ function in the limit $\Delta_{A, M} \rightarrow$ 0 with fixed $\Delta_{V}>0$ is always positive (open circles in Fig. 4), as reported in Refs. [13,14]. This limit is equivalent to the limit $\hbar \omega_{0} / \Gamma \rightarrow 0$ in Eq. (1). Both limits induce a crossover from the unitary class with a topological term to the symplectic class with a topological term [11,12]. This is different from the limit $\hbar \omega_{0} / \Gamma \rightarrow 0$ in the conventional IQHE that realizes a crossover from the unitary class with a topological term to the orthogonal class. The fact that the orthogonal class is always insulating in two dimensions thus causes all critical states to levitate to large $\nu$ as $\hbar \omega_{0} / \Gamma \rightarrow 0[15,16]$.
We conclude by pointing out the interesting possibility that ripples in graphene alone can realize scaling flows to the IQHE plateau transition point even in the absence of a magnetic field; i.e., graphene with long-range disorder is very close to the quantum critical point of the IQHE. This scenario is consistent with recent low-temperature transport measurements of high-mobility graphene samples that imply a temperature independent minimal conductivity $[2,19]$.

This work was supported by the National Science Foundation under Grant No. PHY05-51164 and by Grant-in-Aid for Scientific Research from MEXT (Grant No. 16GS0219).

[1] A. K. Geim and K.S. Novoselov, Nat. Mater. 6, 183 (2007).

[2] K. S. Novoselov et al., Nature (London) 438, 197 (2005).

[3] Y. Zhang et al., Nature (London) 438, 201 (2005).

[4] The Quantum Hall Effect, edited by R. E. Prange and S. M. Girvin (Springer, New York, 1987).

[5] S. Deser, R. Jackiw, and S. Templeton, Ann. Phys. (N.Y.) 140, 372 (1982).

[6] A. W. W. Ludwig et al., Phys. Rev. B 50, 7526 (1994).

[7] P. M. Ostrovsky, I. V. Gornyi, and A.D. Mirlin, arXiv:0712.0597.

[8] H. Suzuura and T. Ando, Phys. Rev. Lett. 89, 266603 (2002); E. McCann et al., ibid. 97, 146805 (2006); I. L. Aleiner and K. B. Efetov, ibid. 97, 236801 (2006); A. Altland, ibid. 97, 236802 (2006).

[9] D. N. Sheng, L. Sheng, and Z. Y. Weng, Phys. Rev. B 73, 233406 (2006).

[10] M. Koshino and T. Ando, Phys. Rev. B 75, 033412 (2007).

[11] P. M. Ostrovsky, I. V. Gornyi, and A. D. Mirlin, Phys. Rev. Lett. 98, 256801 (2007).

[12] S. Ryu, C. Mudry, H. Obuse, and A. Furusaki, Phys. Rev. Lett. 99, 116601 (2007).

[13] J. H. Bardarson, J. Tworzydlo, P. W. Brouwer, and C. W. J. Beenakker, Phys. Rev. Lett. 99, 106801 (2007).

[14] K. Nomura, M. Koshino, and S. Ryu, Phys. Rev. Lett. 99, 146806 (2007).

[15] T. Ando, J. Phys. Soc. Jpn. 52, 1740 (1983); 53, 3101 (1984); 53, 3126 (1984); 55, 3199 (1986).

[16] D. N. Sheng and Z. Y. Weng, Phys. Rev. Lett. 80, 580 (1998); D. N. Sheng, Z. Y. Weng, and X. G. Wen, Phys. Rev. B 64, 165317 (2001).

[17] D. E. Khmelnitskii, Phys. Lett. A 106, 182 (1984); R. B. Laughlin, Phys. Rev. Lett. 52, 2304 (1984).

[18] K. Nomura and A. H. MacDonald, Phys. Rev. Lett. 96, 256602 (2006); 98, 076602 (2007); T. Ando, J. Phys. Soc. Jpn. 75, 074716 (2006).

[19] Y.W. Tan et al., Eur. J. Phys. Special Topics 148, 15 (2007).

[20] J. W. McClure, Phys. Rev. 104, 666 (1956).

[21] S. V. Morozov et al., Phys. Rev. Lett. 97, 016801 (2006).

[22] A. F. Morpurgo and F. Guinea, Phys. Rev. Lett. 97, 196804 (2006). 